\title{
Letrozole Regimen
}

National Cancer Institute

\section{Source}

National Cancer Institute. Letrozole Regimen. NCI Thesaurus. Code C160078.

A hormone therapy regimen consisting of letrozole that may be used in the treatment of breast cancer. 